\title{
CITOGENÉTICA EM CINCO ESPÉCIES ORNAMENTAIS DE LILIALES
}

\author{
CYTOGENETICS OF FIVE ORNAMENTAL SPECIES OF LILIALES
}

\author{
Marlene Terezinha Lovatto ${ }^{1} \quad$ Alice Battistin $^{2}$
}

\section{RESUMO}

\begin{abstract}
Metáfases mitóticas e núcleos interfásicos foram analisados em cinco espécies ornamentais da ordem Liliales. Lilium longiflorum $(2 n=2 x=24)$, Hemerocallis flava $(2 n=2 x=22)$ e Hemerocallis fulva var. Kwanso $(2 n=3 x=33)$ pertencem a familia Liliaceae. Crinum crispum $(2 n=2 x=22) e$ Hippeastrum parodii $(2 n=4 x=44)$ pertencem a familia Amaryllidaceae. As diferenças observadas entre as espécies foram: número de cromossomos metacêntricos, submetacêntricos, subtelocêntricos e telocêntricos; número e posição das constrições secundárias; tamanho dos cromossomos; comprimento total dos cromossomos; número máximo de nucléolos por núcleo interfásico e porcentagem de nucléolos fusionados.
\end{abstract}

Palavras-chave: espécies-ornamentais, metáfases, cromossomos, cariótipos, nucléolos.

\section{SUMMARY}

Mitotic metaphases and interphase nuclei were analyzed in five ornamental species of Liliales order. Lilium longiflorum $(2 n=2 x=24)$, Hemerocallis flava $(2 n=2 x=22)$ and Hemerocallis fulva var. Kwanso $(2 n=3 x=33)$ are Liliaceae. Crinum crispum $(2 n=2 x=22)$ and Hippeastrum parodii $(2 n=4 x=44)$ are Amaryllidaceae. The differences observed among the species were: metacentric, submetacentric, subtelocentric and telocentric chromosome numbers; number and position of secondary constrictions; chromosome size; total length of chromosomes; maximum number of nucleolus by interphasic nucleus and percentage of fusioned nucleolus.

Key words: ornamental-species, metaphases, chromosome, caryotypes, nucleolus.

\section{INTRODUÇÃO}

A ordem Liliales é constituída por várias famílias entre as quaiš Liliaceae e Amaryllidaceae. A maioria de suas plantas são ornamentais, de origem muito antiga. Atualmente são encontradas nos mais diversificados ambientes no novo e no velho mundo.

A constituição cromossômica da ordem Liliales é razoavelmente complexa. VIJAYAVALLI \& MATHEW (1990), chamaram atenção que a determinação de um provável ancestral com número cromossômico básico certo, é impossível. A diferenciação dos gêneros deverá ter ocorrido a partir de uma gradual acumulação de pequenas mudanças estruturais e numéricas nos cromossomos, bem como mudanças na quantidade de DNA e no número de cromossomos nucleolares.

O gênero Lilium L. com aproximadamente 100 espécies, mantém seu número básico $\mathrm{n}=\mathrm{x}=12$ (DARLINGTON \& WYLIE, 1955; SEN, 1978). Entretanto, variações estruturais com trocas inter $\mathrm{e}$ intracromossômicas e variações no número de cromossomos nucleolares, foram observadas por LANGER \& KOUL (1983); KOUL et al. (1988); VIJAYAVALLI \& MATHEW (1990) e NODA (1991).

Plantas do gênero Hemerocallis L., eram usadas pelos antigos chineses na alimentação e na

\footnotetext{
${ }^{1}$ Engenheiro Agrônomo, Mestre, Professora, Departamento de Biologia, Faculdade de Cruz Alta, RS, Brasil.

2

${ }^{2}$ Naturalista, Doutora em Agronomia, Professora Titular, Departamento de Biologia, Centro de Ciências Naturais e Exatas, Universidade Federal de Santa Maria, 97105-900, Santa Maria, RS. Autor para correspondência. 
medicina, antes do desenvolvimento da escrita (HU, 1968; ZADOO et al., 1975). Este gênero possui como número básico $\mathrm{n}=\mathrm{x}=11$ cromossomos. Hemerocallis fulva, foi uma das espécies mais cultivadas a partir do século passado, por possuir diplóides e triplóides de grande valor ornamental (XIAOBAI, 1986; ZADOO \& NARAIAN, 1987).

Os dois centros de maior distribuição da família Amaryllidaceae são América do Sul e África (ARROYO \& CUTLER, 1984). As plantas do gênero Crinum propagam-se principalmente por via vegetativa e são cultivadas como ornamentais de grande valor (LAKSHMI, 1980). O número básico de cromossomos é $\mathrm{n}=\mathrm{x}=11$ (SHARMA \& BHATTACHARYA, 1956). Segundo RAINA (1978), as espécies diferem muito entre si no número e posições das constrições secundárias. Para NARANJO \& POGGIO (1988), $70 \%$ das espécies do gênero Hippeastrum, são diplóides com número básico de cromossomos $\mathrm{n}=\mathrm{x}=11$, ocorrendo também triplóides, tetraplóides, pentaplóides e hexaplóides.

Este trabalho visou obter informações básicas dos cariótipos e do número de nucléolos por núcleo interfásico, em três espécies da família Liliaceae e duas espécies da família Amaryllidaceae, pelo fato das mesmas serem ornamentais bastante comuns em nosso meio.

\section{MATERIAIS E MÉTODOS}

Da família Liliaceae as três espécies estudadas foram: Lilium longiflorum Thumb., Hemerocallis flava L. e Hemerocallis fulva var. Kwanso Regel., coletadas no ano de 1993, no campus da Universidade Federal de Santa Maria, Santa Maria, RS, Brasil. Da família Amaryllidaceae as espécies estudadas foram: $\boldsymbol{C r}$ num crispum Gouws e Hippeastrum parodii $\mathrm{Huz} \&$ Coc., coletadas em 1993, no bairro Camobi, município de Santa Maria, RS, Brasil.

Cinco exemplares de cada espécie foram coletados e colocados em recipientes com água para emitirem novas raízes. Quando estas atingiram aproximadamente $5 \mathrm{~mm}$ foram pré-tratadas em água destilada numa temperatura de $2^{\circ} \mathrm{C}$ por 24 horas, fixadas em etanol : ácido acético $(3: 1)$ durante 36 horas, transferidas para álcool
$70 \%$ e estocadas na geladeira até o uso. As pontas das raízes foram hidrolisadas em $\mathrm{HCl} 1,0 \mathrm{~N}$ por oito minutos à temperatura de $60^{\circ} \mathrm{C}$. Os corantes usados foram feulgen e orceína lacto-propiônica. A nomenclatura usada na descrição morfológica dos cromossomos foi de LEVAN et al. (1964). Para a estimativa do índice de assimetria intracromossômica $\left(\mathrm{A}_{1}\right)$ e assimetria intercromossômica $\left(\mathrm{A}_{2}\right)$ seguiu-se a metodologia de ZARCO (1986).

Idiogramas: com o valor médio do comprimento total dos cromossomos, em 10 metáfases escolhidas ao acaso, dentro de cada amostra em cada espécie, foram construídos os idiogramas, com o auxílio do programa de computador Fantasy 2.

Coloração dos nucléolos: os processos de pré-tratamento, fixação e hidrólise foram os mesmos usados para os cariótipos. Após as pontas das raízes foram tratadas com 2X SSC (cloreto de sódio e cirtrato de sódio) por cinco minutos a $50^{\circ} \mathrm{C}$, lavadas com água destilada e coradas com $\mathrm{AgNO}_{3}$ (nitrato de prata) $50 \%$ $(\mathrm{m} / \mathrm{V})$. Os preparados foram cobertos com tela de "Salt-nylon" 20 x 20mm (STACK, 1991), incubados a $60^{\circ} \mathrm{C}$ em câmara úmida por aproximadamente duas horas (tempo suficiente para obter a cor desejada).

\section{RESULTADOS E DISCUSSÃO}

$\mathrm{Na}$ Tabela 1 estão registrados os dados referentes às características cromossômicas das cinco espécies estudadas e na Tabela 2 encontram-se os resultados do número máximo de nucléolos por núcleo interfásico, bem como a porcentagem de núcleos com nucléolos fusionados e o total de núcleos interfásicos observados em cada espécie. Metáfases mitóticas e o número máximo de nucléolos por núcleo interfásico

\begin{tabular}{|c|c|c|c|c|c|c|c|c|}
\hline Espécies & $2 n$ & Cariótipo & $\mathrm{X}$ & Satélite* & CTC & IC & $\overline{A_{1}}$ & $\overline{A_{2}}$ \\
\hline L. longiflorum & 24 & $2 m+2 s m+4 s t+16 t$ & 11,97 & N.V. & 287 & 14 & 0,81 & 0,24 \\
\hline H. flava & 22 & $10 m+8 s m+2 s t+2 t$ & 5,00 & BC-10 micro & 111 & 33 & 0,50 & 0,20 \\
\hline $\begin{array}{l}\text { H. fulva var. } \\
\text { Kwanso }\end{array}$ & 33 & $15 m+9 s m+6 s t+3 t$ & 4,91 & BC-9 micro & 162 & 34 & 0,47 & 0,24 \\
\hline C. crispum & 22 & $8 m+10 s m+4 s t$ & 10,71 & BL-10 micro & 236 & 34 & 0,48 & 0,28 \\
\hline H. parodii & 44 & $16 m+16 s m+12 s t$ & 8,65 & N.V. & 380 & 28 & 0,58 & 0,32 \\
\hline
\end{tabular}




\begin{tabular}{|c|c|c|c|c|c|c|c|c|c|}
\hline \multirow{2}{*}{ Espécies } & \multirow{2}{*}{$\begin{array}{l}\text { Células } \\
\text { estudadas }\end{array}$} & \multirow[b]{2}{*}{1} & \multicolumn{6}{|c|}{ Combinações de fusão (\%) } & \multirow[b]{2}{*}{8} \\
\hline & & & 2 & 3 & 4 & 5 & 6 & 7 & \\
\hline \multirow{5}{*}{$\begin{array}{l}\text { L. longiflorum } \\
\text { H. flava } \\
\text { H. fulva var. } \\
\text { Kwanso } \\
\text { C. crispum } \\
\text { H. parodii }\end{array}$} & 414 & 02 & 30 & 20 & 35 & 12 & 01 & -- & \\
\hline & 744 & 26 & 31 & 26 & 10 & 06 & 01 & - & \\
\hline & 648 & 07 & 12 & 22 & 21 & 13 & 16 & 05 & 04 \\
\hline & 1045 & 80 & 20 & $\overline{-r}$ & 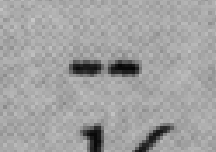 & - & -- & -- & - \\
\hline & 301 & 12 & 33 & 39 & 16 & - & -- & - & \\
\hline
\end{tabular}

menores que os demais cromossomos do cariótipo (Figura 2E). Segundo GREIZERSTEIN \& NARANJO (1987), cromossomos pequenos ou medianos são comuns nos cariótipos supostamente mais primitivos da família Amaryllidaceae e presentes em espécies de Hippeastrum e Rhodophiala.

Os dados referentes à simetria, mostram que o cariótipo de $\boldsymbol{L}$. longiflorum é o mais assimétrico ( $\mathrm{IC}=14$ e $\mathrm{A}_{1}=0,81$ ). Os outros 4 cariótipos possuem IC (Tabela 1) mais próximo a 50 e $A_{1}$ mais afastado

das espécies, encontram-se na Figura 1 e, seus respectivos idiogramas na Figura 2.

Da família Liliaceae são diplóides: $\boldsymbol{L}$. longiflorum (Figuras: 1a e 2A) com $2 \mathrm{n}=2 \mathrm{x}=24 \mathrm{e}$ Hemerocallis flava (Figuras: 1c e 2B) com $2 n=2 x=22$ e um triplóide Hemerocallis fulva var. Kwanso (Figuras 1e e 2C) $\operatorname{com} 2 n=3 x=33$. As três espécies possuem cromossomos metacêntricos, submetacêntricos, subtelocêntricos e telocêntricos variando apenas no número dos mesmos.

As duas espécies da família Amaryllidaceae, o diplóide Crinum crispum com $2 \mathrm{n}=2 \mathrm{x}=22$ (Figuras 1g e 2D) e o tetraplóide Hippeastrum parodii com $2 n=4 x=44$ (Figuras 1i e 2E) possuem três tipos de cromossomos: metacêntricos, submetacêntricos e subtelocêntricos, constituindo uma característica diferencial entre os exemplares das duas famílias. Chama atenção, o fato de serem encontrados com bastante freqüência exemplares poliplóides nas duas famílias, predominando triplóides na família Liliaceae e tetraplóides na família Amaryllidaceae.

A média do tamanho dos cromossomos (Tabela 1) foi mais alta em $\boldsymbol{L}$. longiflorum $(11,97 \mu)$, seguida de Crinum crispum (10,71 $\mu)$, Hippeastrum parodii $(8,65 \mu)$. As duas espécies do mesmo gênero Hemerocallis flava e Hemerocallis fulva, mantiveram suas médias mais próximas $5,0 \mu$ e $4,9 \mu$ respectivamente. Ocorreu uma acentuada diferença nas médias do comprimento dos cromossomos entre os exemplares dos gêneros na família Liliaceae.

Os pares metacêntrico e submetacêntrico do L. longiflorum (Figura 2A) e o par metacêntrico do Crinum crispum (Figura 2D), são maiores que os demais pares do conjunto. A diferença no tamanho dos cromossomos citados é característico nos dois gêneros. Para VIJAYAVALLI \& MATHEW (1990), essas diferenças de tamanho provavelmente surgiram devido a translocações intracariotípicas. Os cromossomos metacêntricos de Hippeastrum parodii são
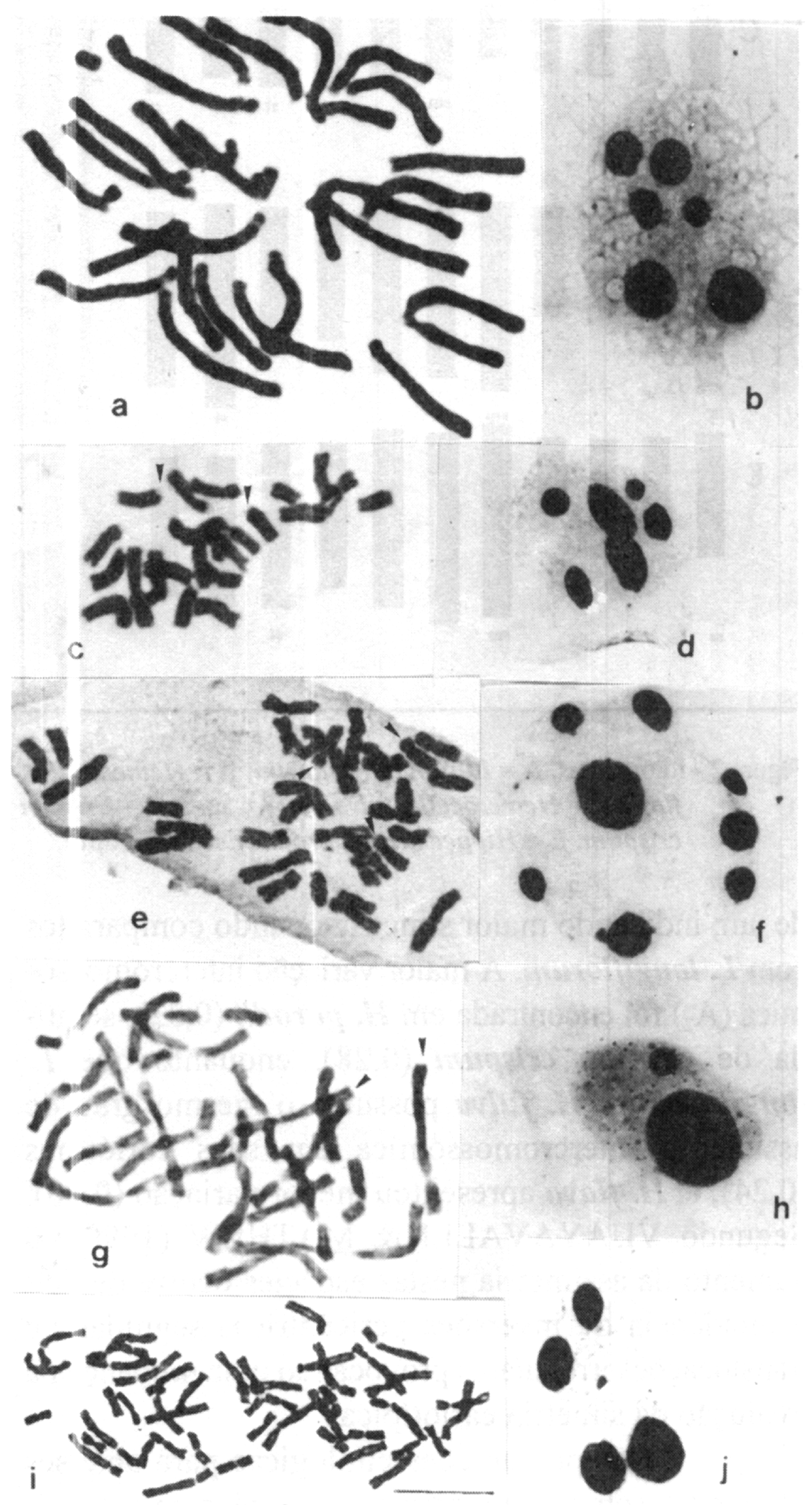

Figura 1 - Metáfises mitóticaṣ e número máximo de nucléolos por núcleo interfásico: $a-b$ Lilium longiflorum $(2 n=2 x=24)$; c-d Hemerocallis flava $(2 \mathrm{n}=2 \mathrm{x}=22)$; e-f Hemerocallis fulva var. Kwanso $(2 \mathrm{n}=3 \mathrm{x}=33)$; g-h Crinum crispum $(2 n=2 x=22)$; $i-j$ Hippeastrum parodii $(2 n=4 x=44)$. As setas indicam satélites. Escala $=10 \mu \mathrm{m}$. 


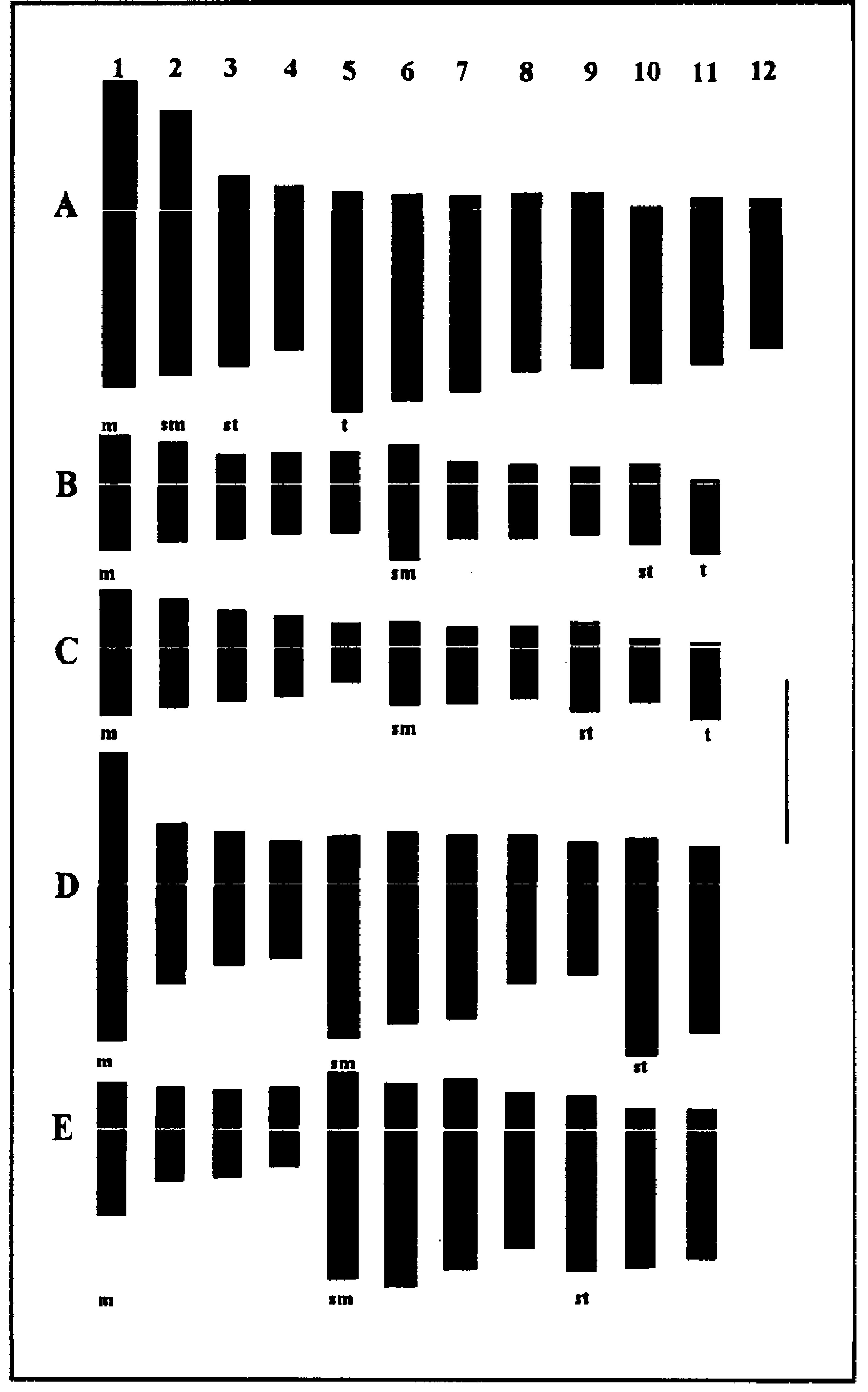

Figura 2 - Idiogramas. $\mathrm{A}=$ Lilium longiflorum $; \mathrm{B}=$ Hemerocallis flava $; \mathrm{C}=$ Hemerocallis fulva var. Kwanso; $\mathrm{D}=$ Crinum crispum $; \mathrm{E}=$ Hippeastrum parodii . Escala $=10 \mu \mathrm{m}$.

de um, indicando maior simetria quando comparados com $\boldsymbol{L}$. longiflorum. A maior variação intercromossômica $\left(\mathrm{A}_{2}\right)$ foi encontrada em $\boldsymbol{H}$. parodii $(0,32)$, seguida de Crinum crispum $(0,28)$, enquanto que $\boldsymbol{L}$. longiflorum e $\boldsymbol{H}$. fulva possuem o mesmo grau de assimetria intercromossômica em seus cariótipos $(0,24)$, e, $\boldsymbol{H}$. flava apresentou menor variação $(0,20)$. Segundo VIJAYAVALLI \& MATHEW (1990), o aumento da assimetria nestas espécies ocorre devido a incidência de inversões pericêntricas seguidas de translocações irregulares provocando uma mudança na evolução da simetria cariotípica.

Nas preparações citológicas para análises dos cromossomos metafásicos, com a técnica convencional (feulgen), não foram visualizadas constrições secundárias em $\boldsymbol{L}$. longiflorum e $\boldsymbol{H}$. parodii, apenas duas em $\boldsymbol{H}$. flava e três em $\boldsymbol{H}$. fulva var. Kwanso e em Crinum crispum. Porém quando células interfásicas foram coradas com $\mathrm{AgNO}_{3}$ observou-se a presença de no máximo seis nucléolos em $\boldsymbol{L}$. longiflorum (Tabela 2 e Figura 1b) e $\boldsymbol{H}$. flava (Tabela 2 e Figura 1d), oito nucléolos em $\boldsymbol{H}$. fulva var. Kwanso (Tabela 2 e Figura 1f), dois nucléolos em $\boldsymbol{C}$. crispum (Tabela 2 e Figura 1 h) e quatro em $\boldsymbol{H}$. parodii (Tabela 2 e Figura $1 \mathrm{j}$ ), indicando a presença de seis, oito, dois e quatro regiões organizadoras nucleolares (RONs) respectivamente nas espécies citadas. Nestas regiões os genes transcricionais estão ativos, caracterizando cada uma das espécies.

O fato de constrições secundárias não terem sido visualizadas em cromossomos metafásicos, indica que podem as mesmas estar localizadas nas extremidades dos cromossomos ou muito próximas ao centrômero, não sendo possível detectá-las com o uso de técnicas convencionais nas análises de cromossomos.

Outro fator que merece destaque é a forte tendência dos nucléolos se fusionarem dentro do mesmo núcleo, durante a interfase (Tabela 2). A tendência de fusão entre os nucléolos do mesmo núcleo, provavelmente deve-se ao fato de estarem ocorrendo as mesmas reações químicas na transcrição do rDNA para rRNA, intensificando a atividade gênica.

Segundo NODA (1991), o número máximo de nucléolos interfásicos decresce com o aumento do grau de especialização do cariótipo. Se esta hipótese for correta, os dois exemplares da família Amaryllidaceae, $\boldsymbol{C}$. crispum e $\boldsymbol{H}$. parodii, estão num grau de especialização mais elevado comparadas com os três exemplares da família Liliaceae: $\boldsymbol{L}$. longiflorum, H. flava e $\boldsymbol{H}$. fulva var. Kwanso, e, esta última a menos especializada.

\section{AGRADECIMENTOS}

Este trabalho foi realizado com apoio do CNPq, FAPERGS e FIPE/UFSM. As autoras agradecem ao Prof. Dr. Reinaldo Simóes Gonçalves o auxílio na montagem do programa no computador para confecção dos idiogramas.

\section{REFERÊNCIAS BIBLIOGRÁFICAS}

ARROYO, S. C., CUTLER, D. F. Evolutionary and taxonomic aspects of the internal morphology in Amaryllidaceae from the South America and Souther Africa. Kew Bulletin, London, $v$. 39, n. 3, p. $467-498,1984$

DARLINGTON, C. D., WYLEI, A. P. Chromosome atlas of flowering plants. London: Allen and Unwin, 1955. $519 \mathrm{p}$

GREIZERSTEIN, E. J., NARANJO, C. A. Estudios cromosomicos en especies de Zephyranthes (Amaryllidaceae). Darwiniana, Tomo, Buenos Aires, v. 28, n. 1-4, p. 169-186, 1987. 
HU, S. Y. Uses of daylily as food and medicine. American hortocultural magazine, Washington, v. 47, n. 2, p. 214-218, 1968.

KOUL, A. K., LANGER. A.; WAFAI, B. A. Studies of nucleolus and nuclear chromosomesin Angiosperms VI. Nucleolar organization in genus Tulipa L. Liliaceae. Cytologia, Tokyo, v. 53 , p. $193-198,1988$.

LAKSHMI, N. Citotaxonomical studies in eight genera of Amaryllidaceae. Cytologia, Tokyo, v. 45, p. 663-673, 1980.

LANGER, A., KOUL, A. K. Studies on nucleolus and nucleolar chromosomes in Angiosperms IX. Lilium Linn. Cytologia, Tokyo, v. 48, p. 519-526, 1983.

LEVAN, A., FREDGA, K., SANDBERG, A. A. Nomenclature for centromeric position on chromosomes. Hereditas, New York, v. 52, n. 2 , p. $201-220,1964$.

NARANJO, C. A., POGGIO, L. A comparasion of karyotype, AgNOR bans and DNA content in Amaryllis and Hippeastrum (Amaryllidaceae). Kew Bulletin, London, v. 43, n. 2, p. 317 325, 1988.

NODA, S. Chromosomal variation and evolution in the Lilium. In: GUPTA, A. K. Developments in plants genetics and breeding $2 B$ chromosome engineering in plants-genetics, breeding, evolution. Elsevier, 1991, part B., p. 507-524.
RAINA, S. N. Genetics mechanisms underlying evolution in Crinum. Cytologia, Tokyo, v. 43, p. 575-580, 1978.

SEN, S. Intraspecific diferentiation in karyotype of Lilium. Cytologia, Tokyo, v. 43, p. 305-315, 1978.

SHARMA, A. K., BHATTACHARYA, N. An investigation on the karyotype of the genus Crinum and its phylogeny. Genetica, Netherlands, n. 28, p. 263-296, 1956.

STACK, M. S. Staining plant cells with silver II. Chromosome cores. Genome, Canada, v. 34, n. 6, p. 900-908, 1991.

VIJAYAVALLI, B., MATHEW, P. M. Cytotaxonomy of the Liliaceae and allied families. India, Continental Publications, Kerala, 1990, $187 \mathrm{p}$.

XIAOBAI, J. The chromosomes of Hemerocallis ( Liliaceae ). Kew Bulletin, London, v. 41, n. 2, p. 379-391, 1986.

ZADOO, S.N., ROY, R. P., KHOSHOO, T. N. Variation in karyotype in Hemerocallis. Cellule, Brussels, v. 71 , p. 253$271,1975$.

ZADOO, S. N., NARAIAN, P. Studies on polymorphism of nucleolar chromosomes in Hemerocallis. Cytologia, Tokyo, v. 52, p. 387-393, 1987.

ZARCO, C. R. A new method for estimating karyotype asymmetry. Taxon, Utrecht, Netherlands, v. 35, n. 3, p. 526-530, 1986.

Ciência Rural, v. 27, n. 4, 1997. 